\title{
El problema interpretativo de la mecánica cuántica. Interpretación minimal e interpretaciones totales*
}

\author{
Alejandro Cassini**
}

\begin{abstract}
Resumen
En este artículo sostengo que la teoría cuántica estándar tiene una interpretación minimal sobre la cual todos los físicos están de acuerdo. Dicha interpretación es suficiente para cualquier aplicación de la teoría y ha sido confirmada por innumerables experimentos. Sin embargo, no proporciona una imagen global del mundo cuántico ni una ontología pretendida para la teoría cuántica. Por esa razón, se han propuesto diversas interpretaciones totales con el fin de completar la interpretación minimal. Argumento, entonces, que dichas interpretaciones, que son empíricamente equivalentes, pero incompatibles entre sí, constituyen empresas metafísicas que no pueden ser confirmadas por ninguna experiencia concebible. Por otra parte, afirmo que el programa de hallar la ontología de la teoría cuántica se basa en el falso supuesto de que existe una única ontología compatible con cada teoría física. Concluyo que no existe ningún camino directo desde el formalismo de la teoría cuántica a una ontología pretendida, ni tampoco desde determinados supuestos metafísicos hacia el formalismo cuántico.

Palabras clave: Teoría cuántica, identificación de teorías, interpretación de teorías, modelos, ontología pretendida.
\end{abstract}

\footnotetext{
* Recibido: octubre 2016. Aceptado: noviembre 2016.

**Consejo Nacional de Investigaciones Científicas y Técnicas / Universidad de Buenos Aires. Buenos Aires, Argentina. Email: alepafrac@yahoo.com.ar
} 


\title{
The Interpretative Problem of Quantum Mechanics. Minimal Interpretation and Total Interpretations
}

\begin{abstract}
In this paper I contend that standard quantum theory has a minimal interpretation, on which all physicists agree. That interpretation is sufficient for every application of quantum theory and it has been confirmed by a countless number of experiments. However, it provides neither an overall picture of the quantum world nor an intended ontology for quantum theory. For those reasons, several full interpretations have been proposed in order to complete the minimal interpretation. I then argue that those interpretations - which are empirically equivalent, but mutually incompatible- are metaphysical ventures that cannot be confirmed by any conceivable experience. Moreover, I claim that the program of finding the ontology for quantum theory rests on the false assumption that there is a unique ontology compatible with each physical theory. I conclude that there is no direct path either from the formalism of quantum theory to its intended ontology, or from determinate metaphysical assumptions to the quantum formalism.
\end{abstract}

Keywords: Quantum theory, theory identity, theory interpretation, models, intended ontology.

\section{Introducción}

La interpretación de la mecánica cuántica ha sido, desde sus mismos orígenes, un problema mal definido. Como se sabe, existen diferentes interpretaciones de la teoría cuántica estándar, ${ }^{1}$ que han sido discutidas extensamente tanto por científicos como por filósofos. Sin embargo, una inspección, aunque sea somera, de la bibliografía existente no permite determinar con pre-

\footnotetext{
${ }^{1}$ Hay una ambigüedad persistente en el uso de la expresión "mecánica cuántica” por parte de científicos y filósofos. A veces se la usa como sinónimo de "física cuántica”, en oposición a la física clásica. Otras veces se la emplea para hacer referencia a una parte o un capítulo de la física cuántica, la mecánica, respecto de la cual podrían existir diferentes teorías rivales. En estos dos casos se la usa como nombre genérico o de clase. Por último, también se la emplea como el nombre propio de una teoría en particular, respecto de la cual se plantea el problema de la interpretación. Aquí no usaré nunca "mecánica cuántica" en el primer sentido. Para referirme a la mecánica cuántica en el último sentido, apelaré la expresión "teoría cuántica estándar” o, a veces, a "mecánica cuántica estándar".
} 
cisión cuántas y cuáles son las interpretaciones que se han propuesto. Tampoco es posible determinar en qué consiste precisamente cada interpretación. Diferentes obras presentan listas diferentes de interpretaciones y, respecto de aquellas que tienen en común, ofrecen caracterizaciones muy distintas. ${ }^{2}$ Como ejemplo de esta situación pueden tomarse dos casos paradigmáticos: la llamada interpretación de Copenhague y la teoría de variables ocultas de David Bohm.

La interpretación de Copenhague la elaboraron principalmente Niels Bohr y Werner Heisenberg desde la época fundacional de la teoría cuántica en 1926-1927. ${ }^{3}$ El nombre mismo sólo apareció a mediados de la década de 1950 y fue popularizado por Heisenberg en su libro Physik und Philosophie, publicado en $1958 .{ }^{4}$ En casi todos los libros de texto sobre mecánica cuántica se menciona la interpretación de Copenhague, pero se la define de maneras muy diferentes, a veces incongruentes entre sí. La causa de esta diversidad se encuentra, seguramente, en la naturaleza a menudo oscura de los escritos de Bohr, que admiten distintas interpretaciones, y en el hecho de que las ideas de Heisenberg y de Bohr siempre fueron bastante diferentes. David Mermin (2004) relata al respecto una anécdota muy ilustrativa. Cuenta que en sus cursos y conferencias solía citar un pasaje de Aage Petersen, un discípulo danés de Bohr, que parecía resumir la esencia misma de la interpretación de Copenhague. ${ }^{5}$ El pasaje es el siguiente:

No hay ningún mundo cuántico. Hay solo una descripción físico-cuántica abstracta. Es incorrecto pensar que la tarea de la física es descubrir cómo es la naturaleza. La física trata acerca de lo que podemos decir sobre la naturaleza. (Petersen, 1963: 12)

\footnotetext{
${ }^{2}$ Resulta imposible, no ya leer, sino siquiera citar, todas las obras que se han dedicado enteramente al problema de la interpretación de la teoría cuántica. Entre las que he podido consultar se encuentran: Bitbol (1996); Bub (1997); Dickson (1998); Friedrichs (2015); Jaeger (2009) y (2014); Jammer (1974); Laloë (2012); Omnès (1994) y (1999); Ruetsche (2011) y Selleri (1994). Esta muestra resulta suficiente para comprobar que no existe acuerdo alguno acerca de qué constituye una interpretación. En ocasiones, el problema ni siquiera se plantea.
}

${ }^{3}$ La Conferencia Solvay de 1927 fue posiblemente el momento más importante. Véase el análisis histórico detallado de Bacciagaluppi y Valentini (2009).

${ }^{4}$ Según Howard (2004), el término lo acuñó Heisenberg en 1955. Desde entonces, las ideas asociadas a esta interpretación han sido principalmente las de Heisenberg mismo más que las de Bohr.

${ }^{5}$ Petersen (1968) presenta sus ideas sobre la filosofía de la mecánica cuántica de manera más detallada. 
En cierta ocasión, Victor Weisskopf, un distinguido físico alemán formado en el Instituto de Bohr en Copenhague, al escuchar esta cita exclamó “¡Bohr nunca podría haber dicho algo como eso!". Mermin retiró, entonces, la cita de sus conferencias, suponiendo que Weisskopf era un intérprete autorizado del pensamiento de Bohr. Tiempo después, se encontró con Rudolf Peierls, otro discípulo alemán de Bohr, y lo consultó sobre el asunto. Al escuchar la cita de Petersen, Peierls respondió: "Sí, esa es exactamente la clase de cosas que a Bohr le gustaba decir". Así pues, dos discípulos directos diferían completamente acerca del pensamiento de su maestro. Mermin extrajo del episodio la siguiente lección:

Concluí que no hay ninguna interpretación de Copenhague de la mecánica cuántica. Hay solo un rango de posiciones físico-cuánticas. (Mermin, 2004: 11)

El examen de los libros de texto confirma esta impresión. No es que no exista algo llamado "interpretación de Copenhague de la mecánica cuántica", sino, más bien, el problema es que esta es una interpretación esencialmente mal definida y presentada en términos sumamente vagos. Se trata, entonces, de un conjunto de interpretaciones que tienen un parecido de familia en común, pero que no concuerdan en muchos aspectos importantes, y no sólo en los detalles. ${ }^{6}$

El otro caso ejemplar es de la teoría de variables ocultas de Bohm. En 1952 Bohm presentó lo que él llamó "una interpretación alternativa de la teoría cuántica en términos de variables ocultas" (Bohm, 1952: 166). La consideró una alternativa a la "interpretación usual" de esta teoría, y con esa expresión se refería a la interpretación de Copenhague (lo cual muestra que este rótulo todavía no estaba en uso). Posteriormente, Bohm la llamó "interpretación causal" (Bohm, 1957: 116) y luego "interpretación ontológica" (Bohm y Hiley, 1993: 2). A pesar de estos cambios en la denominación, parece claro que Bohm no pretendía formular una teoría alternativa a la mecánica cuántica estándar, sino una nueva interpretación de esta teoría. Sin embargo, el propio Bohm en sus escritos también la llama "teoría" y "modelo" (Bohm, 1957: 118-119), lo cual sugiere que no se trata de una mera interpretación, sino de una teoría alternativa. En toda la bibliografía científica y filosófica sobre las variables ocultas se llama a la teoría de Bohm, de manera indistinta, "interpretación", "teoría", "modelo", “enfoque", "punto de vista", "formulación”,

\footnotetext{
${ }^{6}$ Esa misma idea ya había expresada, entre otros, por Jammer (1974).
} 
"versión”, “explicación” y "alternativa", entre otras expresiones. El nombre "Mecánica Bohmiana", con el que se conoce a la formulación más reciente de esta teoría, no permite determinar si se trata de una teoría alternativa a la teoría cuántica estándar o si es una interpretación diferente de esta teoría. ${ }^{7}$ Actualmente, muchos admiten que se trata de una teoría alternativa, aunque empíricamente equivalente, a la mecánica cuántica estándar, pero todavía es frecuente que se la llame "interpretación". ${ }^{8}$ En la práctica, muchos científicos no distinguen entre interpretaciones de una teoría dada y teorías alternativas a ella.

La dificultad que subyace a estos dos problemas es que el concepto mismo de "interpretación de la mecánica cuántica" es sumamente vago y no ha sido satisfactoriamente elucidado. Como consecuencia de ello, no es posible determinar con precisión cómo caracterizar una interpretación determinada de la teoría cuántica estándar, ni tampoco cómo distinguirla de una teoría alternativa. Antes de poder ofrecer una interpretación de una teoría física, ya sea una mecánica cuántica o cualquier otra, es necesario, ante todo, poder identificarla. Luego, debería ser posible determinar por qué dicha teoría necesita una interpretación diferente de la que ya tiene. De manera más general, habría que esclarecer cuáles son las condiciones que debería cumplir una interpretación satisfactoria de la teoría. Para ello, previamente habría que tener en claro qué es lo que puede hacer y qué es lo que no puede hacer una interpretación de una teoría física o de una teoría empírica en general. Sólo así será posible elucidar los problemas que plantean las diferentes interpretaciones de la mecánica cuántica.

Aquí no pretenderé ofrecer una respuesta, ni siquiera provisional, a todas estas preguntas. Simplemente, trataré de esclarecer algunas cuestiones preliminares que permitirán distinguir entre interpretaciones de la teoría cuántica y teorías alternativas. El resultado de esta elucidación será que algunas de las llamadas interpretaciones son teorías alternativas a la teoría cuántica estándar. Luego, distinguiré entre dos clases de interpretaciones de la teoría cuántica estándar, una minimal y otra total. Argumentaré que la interpretación

\footnotetext{
${ }^{7}$ Para las formulaciones más recientes de la teoría véase Dürr y Teufel (2009); Dürr, Goldstein y Zanghi (2013).

${ }^{8}$ Entre quienes la consideran una teoría alternativa se cuentan Albert (1992) y, más explícitamente, Riggs (2009). Muchos filósofos de la ciencia la llaman teoría, pero la incluyen entre las interpretaciones de la mecánica cuántica estándar, como una interpretación alternativa a la de Copenhague (por ejemplo, Cushing 1998, Cap. 23). La mayoría de las obras dedicadas a la teoría de Bohm son ambiguas sobre este punto, por ejemplo, Holland (1993) y Gouesbet (2013).
} 
minimal es indispensable y ampliamente compartida por todos los físicos, mientras que una interpretación total, en principio, es prescindible, y, en cualquier caso, sumamente discutible.

Como primer paso, presentaré de manera muy sintética la noción de interpretación para las teorías formales, que es clara y precisa. Esto servirá como punto de comparación para plantear el problema de la interpretación de las teorías empíricas. Luego, discutiré la cuestión de los criterios de identidad para las teorías empíricas. Finalmente, intentaré formular el problema de la interpretación de la teoría cuántica en términos mejor definidos que los usuales. Aunque estableceré algunos de los límites y las tareas de la interpretación de la mecánica cuántica, no intentaré formular un conjunto completo de condiciones (necesarias y suficientes) que debería satisfacer toda interpretación. Finalmente, discutiré el problema ontológico de las teorías cuánticas. Argumentaré al respecto que la idea de encontrar la ontología de la teoría cuántica estándar, o de cualquier otra teoría alternativa, es una cuestión mal planteada porque se apoya en el supuesto erróneo de que existe una ontología única para cada teoría. Finalmente, formularé dos argumentos generales contra las interpretaciones totales de cualquier teoría cuántica. De estos argumentos concluiré que todas las interpretaciones de esta clase no son contrastables por la experiencia, por lo que necesariamente resultan incursiones en la metafísica, en particular, en un tipo de metafísica que la ciencia no parece necesitar y que la filosofía ha abandonado hace tiempo.

\section{La identificación de la teoría cuántica}

En el campo de las ciencias formales los conceptos de teoría y de interpretación están claramente definidos, por lo que no pueden presentarse dudas acerca de si una supuesta interpretación de una teoría dada es en realidad una teoría alternativa. Además, también están precisamente determinadas las condiciones que debe cumplir cualquier interpretación de una teoría. Por último, el concepto de modelo está bien definido y, por consiguiente, está clara la distinción entre interpretaciones que constituyen un modelo de una teoría dada e interpretaciones que no son modelos de dicha teoría.

En el marco de la teoría de modelos clásica el criterio de identidad de una teoría está bien determinado: una teoría es el conjunto de todos los teoremas de dicha teoría o, más en general, es el conjunto de todas las consecuencias lógicas de un conjunto dado de proposiciones. Este criterio es epistemológicamente neutral, es decir, es independiente de si se tiene una posición realista o anti-realista acerca de las teorías científicas. En segundo lugar, toda inter- 
pretación de una teoría formal está sujeta a ciertas restricciones impuestas por el lenguaje de dicha teoría. La interpretación debe asignar un único significado a cada término primitivo del lenguaje de la teoría, respetando la categoría lógica o gramatical de cada símbolo. En tercer lugar, es claro que los modelos de una teoría están sujetos a una restricción ulterior, mucho más estricta: deben satisfacer simultáneamente todos los axiomas de la teoría (o todas las fórmulas de la teoría, en caso de que no esté axiomatizada).

Finalmente, resulta bien determinado aquello que una interpretación no puede hacer respecto de una teoría dada. No puede cambiar la lógica subyacente de la teoría, no puede cambiar el lenguaje de la teoría, no puede modificar la base axiomática de la teoría ni, por consiguiente, alterar el conjunto de los teoremas de dicha teoría. Cualquier cambio en una teoría que tenga como consecuencia un cambio en el conjunto de los teoremas de esa teoría, necesariamente produce un cambio de teoría. Por ejemplo, si se cambia la lógica subyacente de la teoría, sin modificar su lenguaje ni su base axiomática, es posible que se produzca un cambio de teoría porque algunos teoremas no sean deducibles mediante la nueva lógica. Por otra parte, si se modifica el conjunto de los axiomas, ya sea retirando o agregando algún axioma independiente, o bien reemplazando algún axioma por otro no equivalente a éste, el resultado será necesariamente una teoría diferente, ya que se habrá cambiado el conjunto de los teoremas de la teoría original. En cambio, si se modifica el lenguaje o la base axiomática de una teoría, de modo tal que el resultado sea otra teoría lógicamente equivalente a dicha teoría, se obtiene una reformulación de tal teoría. Una interpretación de una teoría dada no se propone reformularla de ninguna de estas dos formas, ni modificando su lenguaje, ni cambiando su base axiomática.

Las consideraciones anteriores permiten concluir que, cualesquiera sean las condiciones que deba satisfacer una interpretación de una teoría física, como la teoría cuántica estándar, la interpretación no puede consistir en reformular la teoría, por ejemplo, mediante otro formalismo o mediante otros axiomas o postulados, ni tampoco en reemplazar a esta teoría por una teoría alternativa. La reformulación de una teoría puede tener muchas virtudes epistemológicas: puede ser más simple, más clara, más elegante, más económica o más bella, pero no constituye una interpretación de dicha teoría. Por otra parte, es obvio que el reemplazo de una teoría por otra tampoco es una interpretación de la teoría. En la física, el cambio de teoría puede ser necesario tanto por razones lógicas como empíricas, ya sea porque la teoría es inconsis- 
tente o porque es empíricamente inadecuada, pero la determinación de estas propiedades (consistencia lógica y adecuación empírica) no es una cuestión que competa a la interpretación de tal teoría.

Una condición necesaria para la interpretación de una teoría es, ineludiblemente, la identificación de la propia teoría. En el caso de las teorías formales, los criterios de identidad están bien definidos y son ampliamente compartidos, de modo que no pueden surgir dudas acerca de los límites de una teoría dada. En el caso de las teorías empíricas, la identificación de la teoría constituye un auténtico problema. Cuando la teoría en cuestión se encuentra axiomatizada, la teoría puede identificarse con el conjunto de proposiciones que son consecuencias lógicas de los axiomas, a la manera clásica, o alternativamente, como la clase de los modelos de dichos axiomas, como en el estructuralismo metateórico y otras variedades de la llamada concepción semántica de las teorías. Dado que no existe una axiomatización de la teoría cuántica que goce de aceptación entre la comunidad de los físicos, ninguna de estas dos estrategias resulta aplicable en la situación actual.

Algunos filósofos de la ciencia, como Ronald Giere (1988), han sostenido que las teorías empíricas son entidades esencialmente vagas, cuyos límites son borrosos. Para Giere, este hecho es una consecuencia de su manera de concebir a las teorías como colecciones de modelos relacionados entre sí por una semejanza informal. Dado que la semejanza entre los modelos no es transitiva y se pierde gradualmente, no es posible delimitar de manera precisa cuáles son los modelos que pertenecen a una teoría dada. Si ello es así, la identificación de la teoría no puede hacerse de manera precisa. Pero si la teoría cuántica no pude identificarse con alguna precisión, el problema de la interpretación de dicha teoría resulta insoluble por el simple hecho de que no está claro qué es lo que debe interpretarse.

Muchos libros de texto suelen presentar la teoría cuántica estándar mediante un conjunto de principios, postulados, leyes o reglas fundamentales de la teoría. La manera de formular estos principios varía mucho según la obra. En la mayoría de los casos, la lista es una colección heterogénea de reglas interpretativas y leyes empíricas. Además, tales principios no constituyen una base axiomática suficiente para la axiomatización de la mecánica cuántica. No obstante, permiten delimitar de manera aproximada el alcance de la teoría. ${ }^{9}$

\footnotetext{
${ }^{9}$ Entre las muchas obras que emplean este modo no estrictamente axiomático de presentar la teoría, pero al menos, basado en postulados, se cuentan: Cohen-Tannoudji, Diu y Laloë (1977); Isham (1995) y Weinberg (2013).
} 
Comoquiera que se interprete una teoría empírica, parece claro que la interpretación no debe permitir la derivación de nuevas consecuencias observacionales, ya que en tal caso, el resultado sería una teoría diferente. Al respecto, Bas Van Fraassen señala que:

Supongamos que acordamos que, en principio, puede haber más de una interpretación adecuada de una teoría. Por tanto, se sigue que las interpretaciones van más allá de la teoría; la teoría + la interpretación es lógicamente más fuerte que la teoría misma. (Pues, ¿cómo podría diferenciar entre visiones, todas las cuales aceptan la teoría, a menos que variaran en lo que agregan a ella?) Entonces, una interpretación introduce factores que no se encuentran en la teoría originalmente. ¿Y qué otra cosa significa "variables ocultas"? La superfluidad empírica se requiere para asegurar que no se siguen predicciones nuevas o diferentes, pues, de otro modo, tenemos una teoría alternativa más que una interpretación. (Van Fraassen, 1991: 243-244).

El criterio proporcionado por Van Fraassen para las interpretaciones de una teoría física, el de ser empíricamente superfluas, o mejor, empíricamente equivalentes, puede considerarse como un par de criterios parciales para la identidad y la diferencia de las teorías empíricas en general. Se dice que dos teorías son empíricamente equivalentes cuando: a) tienen consecuencias observacionales, y b) toda consecuencia observacional de una de ellas es también una consecuencia observacional de la otra. Así, las teorías empíricamente equivalentes tienen exactamente las mismas consecuencias observacionales. La equivalencia empírica es condición necesaria de la identidad entre teorías: (1) si $T_{1}=T_{2}$, entonces, $T_{1}$ y $T_{2}$ son empíricamente equivalentes. Por su parte, la no equivalencia empírica es condición suficiente de la diferencia entre teorías: (2) si $T_{2}$ y $T_{2}$ no son empíricamente equivalentes, entonces, $T_{1} \neq T_{2}$.

Estos dos criterios tienen amplia aceptación, a menudo de manera implícita, entre científicos y filósofos de la ciencia. No obstante, sólo proporcionan un criterio parcial para la identificación de una teoría. La conversa de estos dos condicionales, si fuera aceptable, proporcionaría un criterio completo junto con los dos anteriores, es decir, ofrecería condiciones necesarias y suficientes para la identidad y la diferencia de teorías. Sin embargo, las condiciones (3) si $T_{1}$ y $T_{2}$ son empíricamente equivalentes, entonces, $T_{1}=T_{2}$; y (4) si $T_{2} \neq T_{2}$, entonces, $T_{1}$ y $T_{2}$ no son empíricamente equivalentes, sólo son aceptables para una posición estrictamente empirista acerca de las teorías. De acuerdo con esta concepción, la identidad de una teoría está dada por la clase 
de sus consecuencias observacionales. Ha sido una idea frecuente entre los positivistas y empiristas lógicos, y entre los verificacionistas en general. Más recientemente, Quine la suscribió de manera explícita:

Las teorías pueden diferir completamente en los objetos que recorren sus variables de cuantificación, y todavía ser empíricamente equivalentes, [...] Difícilmente parecemos autorizados a llamarlas dos teorías; son dos maneras de expresar una y la misma teoría. (Quine, 1992: 96)

Se sigue de esta concepción que dos teorías empíricamente equivalentes, por mucho que puedan diferir en sus postulados teóricos y en su ontología pretendida, no son más que dos formulaciones diferentes de una y la misma teoría. Los filósofos y científicos de inclinación realista, o antipositivista en general, difícilmente puedan aceptar este criterio de identidad para las teorías. Se trata de un criterio que no es epistemológicamente neutral y que no puede, por tanto, aceptarse sin mayores reservas.

Con todo, el criterio parcial para la diferencia entre teorías provisto por la condición (2) arroja un resultado esclarecedor para las interpretaciones de la mecánica cuántica. Una supuesta interpretación de la teoría cuántica estándar que no sea empíricamente equivalente a dicha teoría no es una interpretación, sino una teoría alternativa. Esto es lo que ocurre con la teoría del colapso espontáneo de Ghirardi, Rimini y Weber (1986), conocida como GRW y frecuentemente presentada como una interpretación de la mecánica cuántica. Sin embargo, Giancarlo Ghirardi, su principal artesano, reconoce explícitamente que se trata de una teoría rival, o mejor, de una familia de teorías rivales:

[...] los modelos de reducción dinámica producen modificaciones matemáticas precisas de la teoría estándard y como tales, al menos en principio, implican consecuencias físicas precisas que contradicen a la teoría. En este respecto, tales teorías [las de tipo GRW] no constituyen meramente reinterpretaciones de la mecánica cuántica con el mismo contenido empírico, sino teorías genuinamente rivales capaces de ser falsadas en un laboratorio. (Ghirardi, 2005: 425-426)

La teoría GRW no es empíricamente equivalente a la teoría cuántica estándar. Por ejemplo, según GRW la energía no se conserva cuando ocurre un colapso espontáneo del vector de estado. En principio, al menos, es posible diseñar un experimento crucial que contraste ambas teorías respecto de alguna consecuencia observacional en la que difieren. En la práctica, tales 
experimentos no han sido posibles hasta ahora, pero en el futuro la teoría GRW podría ser disconfirmada por alguna experiencia que confirme a la teoría cuántica estándar, como sugiere Ghirardi.

La teoría de variables ocultas de Bohm, en cambio, es empíricamente equivalente a la mecánica cuántica estándar, por lo que este criterio no permite determinar si se trata de una interpretación de esta teoría o de una teoría alternativa. De hecho, como ya hemos señalado, tanto científicos como filósofos de la ciencia han tenido una posición ambigua al respecto. ${ }^{10}$

\section{La formulación de la teoría}

El segundo paso en la interpretación de una teoría empírica consiste en identificar la formulación estándar de la teoría, en particular, el formalismo matemático que se emplea en dicha formulación. En el caso de la teoría cuántica, la formulación estándar se debe principalmente a Dirac (1930) y Von Neumann (1932), que establecieron, respectivamente, la notación y el formalismo actualmente empleados. La manera de presentar este formalismo tiene dos partes. La primera parte consiste en la especificación del espacio de los estados de los sistemas físicos sobre los que trata la teoría. En la formulación estándar, el espacio de los estados es el espacio de Hilbert, un espacio vectorial complejo de infinitas dimensiones. La segunda parte consiste en la presentación de las leyes dinámicas de la teoría, leyes que determinan la evolución temporal de los puntos de ese espacio de los estados. En la teoría cuántica estándar la ley dinámica fundamental es la ecuación de Schrödinger, una ley determinista que especifica cómo evoluciona en el tiempo un sistema físico cerrado, es decir, que no interacciona con su entorno, con otros sistemas físicos. Determina la llamada evolución unitaria de los sistemas físicos, que es continua y, en muchos aspectos, semejante a la de las ondas clásicas, como las ondas electromagnéticas.

Conjuntamente, el espacio de los estados y la dinámica constituyen la estructura de una teoría física. Una interpretación de la teoría cuántica estándar no debería modificar la estructura de dicha teoría. Si lo hace, puede sostenerse que el resultado es una teoría alternativa, incluso aunque sea empíricamente equivalente a la teoría estándar. Esto puede postularse como un criterio general para la identidad de una teoría física: si se modifica el espacio de los estados y/o la dinámica de una teoría física, es decir, alguno de los dos

\footnotetext{
${ }^{10}$ La teoría de Bohm, se ha sostenido, podría no ser empíricamente equivalente a la teoría estándar, pero hasta el momento no se ha encontrado ninguna predicción que no tengan en común.
} 
aspectos de su estructura, se obtiene una teoría alternativa. Este es un criterio independiente del de la equivalencia empírica. Se lo puede llamar el criterio de la conservación estructural. De la aplicación de este criterio se sigue que la teoría de variables ocultas de Bohm, o la Mecánica Bohmiana, no es una interpretación de la teoría cuántica estándar, sino una teoría rival, aunque empíricamente equivalente. La teoría de Bohm mantiene la dinámica de la teoría estándar (es decir, no cambia la ecuación de Schrödinger), pero no conserva el espacio de los estados, sino que lo modifica introduciendo nuevos estados posibles, aquellos representados por las llamadas variables ocultas. De este modo, en la teoría de Bohm el espacio de los estados de la teoría cuántica estándar debe suplementarse con un espacio de los estados para las nuevas variables. Estas variables representan, esencialmente, las posiciones de las partículas que están siempre bien determinadas y, como consecuencia de ello, también lo están sus respectivas trayectorias en el espacio y en el espacio-tiempo.

El criterio de la conservación estructural también arroja el resultado de que la teoría GRW es una teoría alternativa a la mecánica cuántica estándar. En este caso, la teoría mantiene sin cambios el espacio de los estados de la teoría estándar, pero modifica la dinámica lineal de la mecánica cuántica estándar introduciendo un término no lineal en la ecuación de Schrödinger. Como consecuencia del cambio en la ley dinámica fundamental, la teoría GRW tiene consecuencias observacionales diferentes de las de la teoría cuántica estándar. En principio, esta situación se puede generalizar: si una teoría modifica el espacio de los estados de una teoría dada, pero no cambia la dinámica de tales estados, el resultado será una teoría empíricamente equivalente a la primera, donde la trayectoria de un punto en el espacio de los estados será idéntica a la trayectoria que predice la teoría original. En cambio, si la nueva teoría modifica la dinámica de la anterior (de una manera no trivial, es decir, no equivalente), el resultado será una teoría que no es empíricamente equivalente a la primera, una teoría que predice una trayectoria diferente para al menos algunos puntos del espacio de los estados.

En síntesis, tanto la Mecánica Bohmiana como la teoría del colapso espontáneo de GRW (en realidad, una familia de teorías) son teorías alternativas a la mecánica cuántica estándar y no interpretaciones de esta teoría. La teoría GRW resulta una teoría alternativa porque viola las dos condiciones que debería cumplir toda interpretación de una teoría física: la equivalencia empírica y la conservación estructural. La Mecánica Bohmiana, por su parte, cumple con la primera de las condiciones, pero viola la segunda. 
La llamada interpretación de los muchos mundos (many worlds), o del estado relativo, de Everett, en cambio, no es una teoría alternativa según estos criterios, ya que no modifica ni el espacio de los estados ni la dinámica lineal de la teoría cuántica estándar, es decir, deja intacta la totalidad de la estructura de la teoría y, por consiguiente, resulta empíricamente equivalente a la teoría cuántica ortodoxa. David Wallace (2013: 212) la llama una "interpretación pura", pero esta expresión no es particularmente feliz, ya que parece aceptar implícitamente la existencia de interpretaciones "impuras", aunque no es claro que pueda significar esto. Desde el punto de vista que he propuesto aquí, no hay lugar para interpretaciones impuras; o bien una interpretación mantiene sin cambios el espacio de los estados y la dinámica de la teoría cuántica estándar, o bien constituye una teoría alternativa.

\section{La interpretación de la teoría}

La especificación de la estructura de una teoría física (esto es, un espacio de los estados y una dinámica) no es suficiente para formular una teoría física, o, más en general, una teoría empírica. Un formalismo matemático puro carece de contenido empírico y no permite derivar ninguna predicción contrastable. Para obtener una teoría física es necesario que el formalismo tenga una interpretación, al menos parcial, en términos físicos. Por interpretación parcial debe entenderse una que no interpreta la totalidad de los símbolos matemáticos del formalismo, sino solo algunos de ellos. Por interpretación en términos físicos debe entenderse una interpretación, total o parcial, que asigna un significado físico a los símbolos matemáticos. Usualmente esta clase de interpretación correlaciona un símbolo matemático con un significado físico especificando qué clase de entidad física representa ese símbolo matemático (tomando aquí entidad en su sentido más amplio, que incluye, objetos, propiedades, relaciones, eventos y procesos físicos). Si ningún símbolo matemático tiene una interpretación física, la teoría en cuestión es una teoría puramente formal. Esto vale en general para cualquier teoría física. En el caso de la mecánica cuántica, es evidente que un espacio de Hilbert por sí mismo es un objeto matemático del cual no puede deducirse ninguna predicción acerca de ningún fenómeno físico o resultado experimental. Incluso si se le agrega una dinámica a este espacio, como la que proporciona la ecuación de Schrödinger, sólo pueden predecirse trayectorias en el espacio de configuración, pero tales trayectorias son entidades puramente geométricas, meras líneas (o conjuntos de puntos) en un espacio matemático abstracto, que no tienen relación alguna con el comportamiento de las entidades que supuestamente componen el mundo físico. 
Muchos científicos que adoptan una posición instrumentalista respecto de la mecánica cuántica rechazan el problema mismo de la interpretación. Fuchs y Peres (2000), por ejemplo, afirman que la mecánica cuántica "no necesita interpretación" porque sólo proporciona un algoritmo para predecir, de manera probabilista, los resultados de posibles experimentos de medición. Asher Peres proporciona un claro ejemplo de la persistencia de esta posición anti-realista, según la cual "la mecánica cuántica no es una descripción objetiva de la realidad" porque "sólo predice la probabilidad de que ocurran eventos macroscópicos estocásticos" (Peres, 2002: 423). Es muy frecuente encontrar en obras de física afirmaciones según las cuales la mecánica cuántica es un mero formalismo matemático cuya única finalidad es la predicción de resultados experimentales. Los propios Fuchs y Peres expresan esta idea en los siguientes términos.

La teoría cuántica no describe la realidad física. Lo que hace es proporcionar un algoritmo para computar las probabilidades de los eventos macroscópicos (los "clicks en los detectores") que son las consecuencias de nuestras intervenciones experimentales. (Fuchs \& Peres, 2000: 70)

Sin embargo, la idea de un puro algoritmo matemático para calcular las probabilidades de obtener determinados resultados experimentales es en sí misma incoherente. Sólo una teoría física, es decir, un formalismo interpretado, puede hacer tal cosa.

La mecánica cuántica, como toda teoría física, es un formalismo que ya tiene una interpretación en términos físicos. Incluso el más anti-realista de los físicos o de los filósofos debe admitir este hecho. En el caso de la teoría cuántica, existe una interpretación básica del formalismo que es universalmente compartida por todos los físicos y usuarios de la teoría. Se la suele llamar "interpretación minimal", aunque esta expresión también se ha usado con otros significados. ${ }^{11}$ Usualmente, la interpretación minimal del formalismo de la teoría cuántica estándar se presenta mediante un conjunto de principios. Un examen de diferentes libros de texto muestra que algunos de los primeros principios o postulados que se enuncian son en realidad reglas semánticas que proporcionan una interpretación física de los símbolos matemáticos del espacio de Hilbert. Otros principios, en cambio, formulan leyes naturales.

\footnotetext{
${ }^{11}$ Véase, por ejemplo, Jaeger (2009) Cap. 3, para un panorama de diferentes concepciones de la interpretación.
} 
Los principios más frecuentemente empleados (a veces bajo diferentes nombres, como "postulados", "reglas" o "leyes"), son, entre otros, los siguientes:

$P_{I}:$ Un espacio de Hilbert representa un sistema físico determinado.

$P_{2}:$ Un vector (normalizado) de un espacio de Hilbert representa el estado de un sistema físico.

$P_{3}$ : La superposición lineal de dos vectores no nulos de un espacio de Hilbert representa el estado de un sistema físico (este es el llamado principio de superposición).

$P_{4}$ : El producto tensorial de dos espacios de Hilbert representa dos sistemas físicos en interacción.

$P_{5}:$ Un operador hermitiano en un espacio de Hilbert representa un observable (o una propiedad de un sistema físico).

No pretendo ofrecer aquí una formulación rigurosa de los principios de la teoría cuántica. Con todo, es evidente que los enunciados P1-P5 no son enunciados empíricos que puedan ser confirmados o refutados por la experiencia. Más bien, se trata reglas de interpretación, que tienen un carácter convencional. Cada principio correlaciona un tipo de objeto matemático (como espacios, vectores y operadores) con un determinado tipo de entidad física (sistemas físicos, estados y propiedades de esos sistemas). ${ }^{12}$ Desde un punto de vista lógico, son reglas semánticas o interpretativas, pero de ningún modo leyes físicas o naturales. En cambio, un principio como el siguiente expresa una ley natural:

$P_{6}$ : Todo sistema físico cerrado evoluciona en el tiempo de acuerdo con la ecuación de Schrödinger.

Esta es la ley dinámica fundamental de la teoría cuántica estándar y su carácter es semejante al de las leyes de Newton para la mecánica o las leyes de Maxwell para la electrodinámica. Claramente, no se trata de una regla semántica, sino de una hipótesis empírica acerca del comportamiento de los sistemas físicos reales. Como tal, en principio al menos, es susceptible de ser confirmada o disconfirmada por la experiencia, aunque, como ocurre con

\footnotetext{
12 En los tratados de mecánica cuántica se los formula de diferentes maneras, más o menos equivalentes. Aquí los he enunciado de tal modo que primero se indica el símbolo matemático y luego el contenido físico representado por dicho símbolo.
} 
todas las leyes de la física teórica, no tiene contenido empírico por sí misma, sino en conjunción con todo un sistema de hipótesis auxiliares presupuestas. No obstante, es un enunciado de una naturaleza muy diferente de la de los cinco primeros principios.

La principal conexión entre el formalismo de la teoría cuántica y la experiencia está dada por la llamada regla de Born:

$P_{7}$ : El cuadrado del módulo de un vector de estado expresa la probabilidad de encontrar al correspondiente sistema físico en ese estado determinado.

El carácter y la propia formulación de la regla de Born han sido objeto de discusión, pero aquí no intentaré tomar una posición al respecto. Usualmente la regla se formula en términos operacionales, esto es, como si expresara la probabilidad de que una medición encuentre al sistema físico en un determinado estado, y no la probabilidad de que el sistema mismo se encuentre en tal estado (por ejemplo, la probabilidad de que un experimento en el cual se mida la posición de un electrón dé como resultado el valor $\Delta x$, y no la probabilidad de que el electrón se encuentre en la región $\Delta x$ ). No es claro si la regla de Born debe entenderse como un enunciado empírico susceptible de ser disconfirmado por la experiencia (mediante métodos estadísticos). En cualquier caso, este principio es el que permite deducir las predicciones probabilistas características de la mecánica cuántica.

El principio de superposición a veces se considera como una ley empírica, pero no puede serlo por el hecho de que es una consecuencia puramente matemática del carácter lineal de los espacios de Hilbert. Si se admite que la superposición de estados no es una mera ficción útil, sino que representa una determinada situación del mundo físico, el esclarecimiento de este tipo de fenómeno constituye una de las principales dificultades conceptuales de la teoría cuántica estándar. El conocido problema de la medición, por su parte, se sigue del hecho de que nunca se observa un sistema físico en superposición de estados. Toda vez que se efectúa la medición de un observable de un sistema cuántico, como la posición o el spin de un electrón, el resultado es siempre un valor definido de dicho observable. Sin embargo, la teoría cuántica admite que un sistema se encuentre en superposición de estados y evolucione en el tiempo, en ausencia de toda interacción con otros sistemas físicos, manteniéndose en la misma superposición. Aquí hay un desacuerdo entre la teoría y la experiencia, desacuerdo que ha sido uno de los principales motores 
que impulsaron la búsqueda de una teoría alternativa, como la de Bohm o la de GRW, en la cual la superposición no se admite, o bien colapsa de manera casi instantánea en un estado bien definido.

No resulta sencillo presentar de manera completa las reglas semánticas necesarias y suficientes para especificar la interpretación minimal de la teoría cuántica. En cualquier caso, aquí no intentaré hacerlo. Con todo, es claro que principios como $P_{1}-P_{5}$ parecen ser indispensables para esta interpretación, pero no resulta evidente cuáles sean los principios suficientes. ${ }^{13}$ Por otra parte, los términos físicos (como "sistema", "estado" y "observable") que se correlacionan con los símbolos matemáticos necesariamente deben tomarse como primitivos de la interpretación minimal. En la práctica, nunca se intenta definirlos, sino que meramente se los ilustra con ejemplos típicos: un sistema físico, por ejemplo, es una partícula que se mueve dentro de una caja vacía, la posición de la partícula respecto de las paredes de la caja es un observable, y la posición de la partícula en un instante dado es un estado de ese sistema físico.

Sin una interpretación minimal, la teoría cuántica no podría considerarse como una teoría física, ya que quedaría reducida a un puro formalismo matemático sin contenido empírico. La idea de que la mecánica cuántica no necesita interpretación puede reformularse, entonces, en los siguientes términos: la interpretación minimal de la teoría cuántica estándar es necesaria y suficiente para cualquier aplicación de la teoría y para cualquier uso práctico de ella. Esto es, la teoría cuántica no necesita más interpretación que la que proporciona la interpretación minimal. Formulada de esta manera, es una posición razonable, prudente y defendible, que sería reivindicada por muchos científicos y filósofos de inclinación moderadamente empirista.

Si la interpretación minimal de la teoría cuántica es efectivamente suficiente para cualquier uso práctico de la teoría, quienes afirman que esta teoría requiere una interpretación adicional tienen la carga de la prueba y deberían explicar por qué la interpretación minimal no es totalmente satisfactoria y por qué es necesaria una interpretación más completa. Raramente se encuentra en la bibliografía una argumentación detallada al respecto. Ante todo, parece claro que la interpretación minimal es satisfactoria desde el punto de vista práctico, es decir, para las aplicaciones de la teoría. En la práctica no hay

\footnotetext{
13 Por ejemplo, no es claro si las llamadas reglas selección y de superselección forman parte o no de la interpretación minimal. Usualmente, no se las presenta de esa manera y parece razonable suponer que la interpretación minimal no las incluye. Por otra parte, los principios $P_{1}-P_{7}$ no constituyen una base axiomática suficiente para la teoría cuántica estándar.
} 
objeciones contra esta interpretación, que ha permitido un enorme número de contrastaciones y aplicaciones exitosas de la mecánica cuántica estándar. La objeción más recurrente es de tipo teórico y consiste en afirmar que la teoría cuántica estándar, minimalmente interpretada, no es comprensible o inteligible y no permite entender el comportamiento del mundo físico. Muchos físicos y filósofos no van más allá de formulaciones de este tipo, las cuales resultan poco esclarecedoras ya que el concepto mismo de comprensión que presuponen permanece sin elucidar. Si se intenta precisar la objeción, pueden encontrarse dos razones más precisas del carácter insuficiente de la interpretación minimal.

La primera razón es que la interpretación minimal es incompleta. Aquí no debe entenderse que sea incompleta en el sentido de que sea parcial, es decir, que sólo interprete una parte del formalismo matemático. De hecho, es una interpretación completa desde este punto de vista, ya que todos los símbolos específicos del espacio de Hilbert que pueden estar sujetos a interpretación tienen un significado físico asignado por la interpretación minimal. Esta no interpreta los símbolos matemáticos presupuestos, como los que se refieren a las operaciones aritméticas, los números reales y complejos o las derivadas e integrales, entre otros. Estos símbolos no son propios del formalismo de la mecánica cuántica (sino de todas las teorías de la física matemática) y ya se suponen previamente comprendidos. Son parte de la matemática subyacente a la teoría. La interpretación minimal debe considerarse incompleta en el sentido de que no permite responder determinadas preguntas acerca del sentido de las afirmaciones que se derivan de la teoría. Estas preguntas generalmente se obtienen por comparación con otras preguntas análogas que sí tienen respuestas dentro de las teorías de la física clásica. Un ejemplo lo proporciona el concepto mismo de estado de un sistema cuántico. En la mecánica clásica, el estado de un sistema físico, que se supone compuesto por partículas materiales, está dado por las posiciones y los momentos (relativos a un referencial dado) de todas las partículas que lo componen. En la teoría cuántica, en cambio, no es intuitivamente claro qué significa el estado de un sistema, en particular, uno que se encuentra en superposición de estados. Otro ejemplo lo proporcionan las probabilidades. En la física estadística clásica las probabilidades tienen un significado subjetivo o epistémico y, por tanto, las distribuciones de probabilidad sobre los estados de un sistema físico compuesto, formado por muchas partículas, resulta una expresión de nuestra propia ignorancia acerca del estado determinado de cada partícula del sistema. En el caso de la teoría cuántica, el significado de las probabilidades no es evidente, pero se sabe que hay dificultades conceptuales para interpretarlas como en 
las teorías clásicas. Frecuentemente, se consideran cómo probabilidades objetivas, pero es difícil interpretarlas como frecuencias observadas. Muchos filósofos prefieren una interpretación propensivista, pero esta interpretación tiene problemas para satisfacer el cálculo clásico de probabilidades. Además, se sabe que las probabilidades de la mecánica cuántica estándar no satisfacen los axiomas de Kolmogorov para el cálculo clásico de probabilidades. La interpretación subjetivista, o bayesiana, de las probabilidades como grado de creencia también tiene sus partidarios. En principio, el significado de las probabilidades cuánticas es todavía una pregunta abierta, acerca de cuya respuesta no hay un consenso firme entre científicos o filósofos. ${ }^{14}$

La otra razón que puede encontrarse contra la interpretación minimal es que proporciona una interpretación incoherente. Esto no debe entenderse como si la teoría fuera lógica o matemáticamente inconsistente. Si bien no hay, ni se espera que haya, una prueba de la consistencia absoluta de la teoría cuántica, tampoco se ha encontrado, como ocurre con la mayoría de las teorías matemáticas, una auténtica paradoja o inconsistencia formal dentro de la teoría. La incoherencia debe entenderse en relación con la imagen del mundo físico que la teoría proporciona. Esto es, si se admite que la teoría cuántica tiene como fin ofrecer una descripción del mundo físico, entonces, la descripción que se obtiene de ella no es coherente. Como puede suponerse, el problema fundamental proviene de la superposición de estados, que es, en mi opinión, la dificultad conceptual más importante de la teoría estándar. Así, por ejemplo, la teoría permite describir un sistema físico en una superposición de estados de posición, por ejemplo, una partícula que se encuentra en superposición de estados de posición dentro y fuera de una caja. Es fácil preparar una partícula en ese estado, pero no es claro cuál sea el significado físico de esa situación. Puede argumentarse que si esa descripción se toma literalmente, es decir, como si dijera que la partícula se encuentra simultáneamente y en acto dentro y fuera de la caja, resulta incoherente, e incluso lógicamente contradictoria. Otras situaciones incluso más paradójicas se presentan con la superposición de estados de una partícula dentro de dos o más cajas espacialmente separadas. ${ }^{15}$

\footnotetext{
${ }^{14}$ El axioma que no se satisface es el de aditividad, y ello tiene como consecuencia que no se cumplan varios teoremas fundamentales del cálculo clásico (para una presentación elemental véase Auletta, Fortunato y Parisi (2009: 28-31); y Auletta y Wang (2014: Cap. 2). Sobre la interpretación propensivista en general véase Gillies (2000) Cap. 6. Sobre la interpretación bayesiana de las probabilidades cuánticas véase Bub (2007).

${ }^{15}$ La paradoja de las dos cajas fue concebida por Einstein en 1935 y desarrollada por Louis de Broglie en 1959. Véase Cassini (2016) para un análisis detallado de ella.
} 
Una interpretación satisfactoria de la mecánica cuántica debería, entonces, ser una interpretación completa y coherente, es decir, una que no deje preguntas abiertas, por ejemplo, acerca del significado físico de los estados cuánticos superpuestos ni de las probabilidades de la teoría. Se supone, además, que esta clase de interpretación debería proporcionar una imagen coherente del mundo cuántico, algo que la interpretación minimal no hace ni se propone hacer. La falta de una imagen global del mundo cuántico es una de las objeciones más frecuentes a la teoría estándar. En términos filosóficos, suele reformularse la cuestión diciendo que la teoría cuántica estándar no tiene una ontología definida, o de manera equivalente, que no proporciona las condiciones de verdad, o no especifica los hacedores de verdad de la teoría. Se afirma, entonces, que una interpretación plena de la teoría cuántica debería ofrecer tal ontología. Esta es una cuestión que merece considerarse por separado.

\section{La ontología de la teoría}

Muchos científicos y filósofos reducen la cuestión de la interpretación de la teoría cuántica a la de especificar cuál es la ontología de esta teoría. Así, por ejemplo, Van Fraassen (1991) afirma que interpretar una teoría consiste en decir cómo sería el mundo en caso de que la teoría fuera verdadera. De manera más específica, una interpretación debe responder a las preguntas: "¿Bajo qué condiciones es verdadera esta teoría? ¿Cómo dice que es el mundo?" Van Fraassen señala que "estas dos preguntas son la misma" (1991: 242). Entonces, interpretar la teoría cuántica consiste en determinar cuáles son sus condiciones de verdad, y esto equivale a decir cómo sería el mundo en caso de que la teoría fuera verdadera o, más simplemente, cómo es el mundo según la mecánica cuántica. La respuesta a esta pregunta implica, por tanto, determinar cuáles son los "hacedores de verdad" de la teoría, es decir, cuáles son (o serían) los estados de cosas o las situaciones en el mundo que harían verdadera a la teoría. Esto, implica, evidentemente, especificar la ontología de la mecánica cuántica.

Laura Ruetsche (2011) se expresa en términos similares. Sostiene que interpretar una teoría física consiste esencialmente en determinar cuáles son los mundos posibles según dicha teoría. Por mundos posibles debe entenderse aquellos que son física o nomológicamente posibles. Su concepción se resume en los siguientes términos:

Interpretar una teoría física es caracterizar los mundos que son posibles según esa teoría. Pueden distinguirse dos fases de esa interpretación. Una fase 
identifica las estructuras de esa teoría: sus estados, observables y dinámica. La otra caracteriza las situaciones físicas que cuentan como modelos de la teoría así estructurada. La interpretación es un ejercicio de interpretación nómica: las leyes de una teoría guían la caracterización de sus mundos posibles; la interpretación de una teoría es al mismo tiempo una explicación de la noción de posibilidad nomológica aliada con la teoría. (Ruetsche, 2011: 9).

En el caso de la mecánica cuántica, ya sea la teoría estándar o teorías alternativas, como la de Bohm o GRW, la primera fase de la interpretación no ofrece mayores problemas. La primera fase consiste, simplemente, en identificar la teoría, o, más precisamente, aquello que he llamado su estructura. El espacio de los estados, los observables y la dinámica de cada teoría están perfectamente identificados en cada caso. Para la teoría cuántica estándar, el espacio de los estados es el espacio de Hilbert, los observables son tanto magnitudes clásicas (posición, momento, energía) como específicamente cuánticas (spin) y la dinámica la proporciona la ecuación de Schrödinger. Usualmente, como ya señalé, la estructura de la teoría se caracteriza mediante postulados como $P_{1}-P_{7}$. Sin embargo, la segunda fase de la interpretación ha resultado hasta el momento imposible de realizar; nadie ha podido determinar cuáles son los modelos de la estructura así caracterizada, o, lo que es equivalente, cuáles son los mundos físicamente posibles según esta teoría. En realidad, la primera fase es la identificación de la teoría, por lo que no es parte de la interpretación, sino una condición necesaria de ella. La segunda fase es la interpretación propiamente dicha, para la cual deberían especificarse las condiciones de adecuación. ${ }^{16}$

El supuesto básico de todas las posiciones (que podemos llamar realistas) que consideran que el problema de la interpretación de la mecánica cuántica consiste en determinar cuál es la ontología de la teoría estándar es el de la unicidad de la ontología. De acuerdo con este supuesto, generalmente no explicitado, la relación entre una determinada teoría física y una ontología es unívoca, es decir, una relación de uno a uno. Se supone, entonces, que una teoría física tiene un único referente o, más precisamente, que la teoría determina una ontología específica. Dicha ontología estaría formada por todos los hacedores de verdad de la teoría. Entonces, para desvelar la ontología subya-

\footnotetext{
${ }^{16}$ Ruetsche (2011: 10-12) intenta proporcionar algunas condiciones suficientes de adecuación, pero la formulación que ofrece de ellas resulta más bien vaga. Por ejemplo, la siguiente: "Una interpretación debería permitir a la teoría que interpreta pagar sus deudas explicativas" (to discharge its explanatory duties) (p. 11). No es en absoluto claro cuáles serían esos deberes explicativos que debería cumplir la interpretación de una teoría cuántica.
} 
cente a la teoría cuántica estándar, sería suficiente establecer los referentes de sus términos descriptivos y, a partir, de ellos, los de todos los enunciados que componen la teoría.

El supuesto de la unicidad de la ontología de las teorías físicas encuentra un obstáculo insuperable en la tesis de la relatividad ontológica, tesis que se encuentra fundada en propiedades metalógicas bien establecidas de las teorías en general. Quine (1968), como es bien conocido, popularizó esta tesis y le dio su denominación característica. No obstante, la tesis es sumamente general y no depende de tesis específicas de la filosofía de Quine, como la inescrutabilidad de la referencia, el holismo epistemológico o la subdeterminación de las teorías por la experiencia. Para establecerla, en mi opinión, basta la teoría de modelos clásica, que se puede aplicar a cualquier teoría, ya sea formulada en un lenguaje formalizado o no.

Es un hecho bien establecido, por razones puramente lógicas, que toda teoría satisfacible tiene múltiples modelos, generalmente un número infinito de ellos, independientemente de que lleguemos a conocer siquiera uno. Además, toda teoría de primer orden tiene siempre modelos no estándar, es decir, modelos que no son isomorfos con el modelo pretendido o estándar de la teoría. Por esa razón, ninguna teoría de primer orden es categórica. En razón de la llamada versión ascendente del teorema de Löwenheim y Skolem, si una teoría de primer orden tiene un modelo infinito contable, también tiene un modelo no contable, es decir, uno cuyo dominio es un conjunto que tiene una cardinalidad mayor que la del conjunto de los números naturales. En consecuencia, si una teoría de primer orden tiene un modelo en el dominio de los números naturales, como ocurre en el modelo estándar de la Aritmética de Peano, también tendrá otros modelos en dominios como el de los números reales, o el de los puntos del continuo, y posiblemente muchos otros que no son isomorfos entre sí.

En general, toda teoría, sea o no categórica, tendrá una multiplicidad de modelos en dominios diferentes de objetos. Los axiomas de la teoría restringen la clase de los modelos posibles de dicha teoría, pero no determinan ningún modelo en particular. Incluso en el caso de teorías categóricas, éstas tendrán modelos en dominios de objetos tanto abstractos como concretos. Los axiomas de la teoría, por tanto, no determinan los objetos a los que se refiere la teoría. Al contrario, son siempre compatibles con diferentes clases de objetos, independientemente del dominio particular de objetos a los que la teoría pretenda referirse. Por ejemplo, una teoría física que quiera referirse a partículas materiales que se mueven en el espacio también podrá tener un modelo en el dominio de los números naturales. Se trataría de un modelo no 
pretendido, sin duda, pero, desde un punto de vista puramente lógico, éste es un modelo más entre otros posibles y no tiene ningún privilegio más que el que le pueda conceder la intención de los agentes que lo construyen o lo aplican. En matemática, por otra parte, la mayor parte de las teorías no tiene un modelo pretendido. La teoría de grupos constituye un buen ejemplo. Esta teoría surgió desde un comienzo como una teoría del álgebra abstracta sin aplicación pretendida alguna, y luego se le encontraron modelos (hipotéticos) en dominios muy diferentes de objetos, tales como los números enteros, los cristales y las partículas elementales.

La situación no es esencialmente diferente para las teorías físicas. Suponiendo que una teoría física tenga un modelo pretendido o estándar, también tendrá otros modelos en diferentes dominios. Podría objetarse que una teoría física no es una teoría formal que admita diferentes interpretaciones, sino que es una teoría interpretada desde su mismo origen y que, por tanto, tiene una ontología pretendida. Es indudablemente cierto, como ya argumenté en la sección anterior, que una teoría física cuyo formalismo matemático no esté interpretado no es una teoría empírica, pero de allí no se sigue que sólo tenga una sola interpretación posible. Mucho menos, que la ontología de la teoría sea unívoca. Lo que suele denominarse la ontología de una teoría física solo es la ontología pretendida de la teoría, esto es, la ontología del modelo pretendido de dicha teoría. Por otra parte, al igual que las teorías matemáticas, no toda teoría física tiene un modelo pretendido ni, por consiguiente, una ontología pretendida. La teoría física sólo necesita una interpretación minimal para adquirir contenido empírico, pero esa interpretación minimal puede ser insuficiente para especificar una ontología completa, o incluso coherente, como ocurre en el caso de la teoría cuántica estándar.

La posición filosófica que acepta una "ontología primitiva" para las teorías físicas parece admitir que esta no es otra cosa que una ontología pretendida. Así, Valia Allori escribe que:

La física trabaja por medio de la matemática: una teoría contiene varios objetos matemáticos, algunos con significación física, otros sin ella. El punto aquí es que esto se establece una vez que se propone la teoría: ya hay una interpretación natural para cada objeto matemático, a saber, aquella que el que propone la teoría pretende darle. La elección del científico de lo que existe físicamente en el mundo determinará más o menos automáticamente el objeto matemático para representarlo. [...] Una vez que el científico construye la teoría, la imagen metafísica que ésta proporciona ya ha sido definida y hay una muy limitada libertad para reinterpretar el formalismo. (Allori, 2013: 63) 
La tesis aquí formulada parece suscribir a la vez dos tesis inconsistentes entre sí: una según la cual la ontología de una teoría es aquella que pretenden darle los científicos que la construyen, y otra según la cual esa ontología es única. La primera tesis es cierta, pero la segunda es errónea. El formalismo matemático de una teoría física no determina una interpretación física unívoca para los símbolos matemáticos del formalismo de dicha teoría. Dada una asignación de significado a estos símbolos, por razones puramente lógicas es posible construir una función que reinterpreta de manera uniforme y coherente todos esos símbolos modificando su referencia $y$, por tanto, produciendo una interpretación diferente. Quine (1992: 31-33) la ha llamado una "función proxy" y ha ofrecido ejemplos de cómo opera dicha función. Por otra parte, una ontología pretendida tampoco determina el formalismo matemático con el cual representarla, sino que es compatible con diferentes formalismos. No existe ningún camino unívoco que vaya del formalismo de una teoría física a su ontología pretendida, ni tampoco a la inversa, de la ontología al formalismo.

Los partidarios de la ontología primitiva han admitido, además, que no toda teoría tiene una ontología pretendida. En palabras de la propia Allori:

De hecho, la Mecánica Bohmiana es una teoría con una ontología primitiva: hay partículas (la ontología primitiva) cuya evolución temporal está gobernada por una función de onda de Schrödinger que evoluciona (la variable no primitiva). [...] La teoría GRW [en cambio] no tiene una ontología primitiva. (Allori, 2013: 69)

Incluso admitiendo que la ontología pretendida de la teoría de Bohm es una ontología de partículas materiales que se mueven con trayectorias determinadas en el espacio físico tridimensional (o en el espacio-tiempo cuadridimensional), es seguro que esa teoría es compatible con una ontología en la que no existen las partículas. Por lo demás, muchas teorías físicas no tuvieron en su origen una ontología pretendida y, precisamente por esa razón, resultaron luego compatibles con diferentes teorías posteriores. La relatividad especial, tal como la formuló Einstein en 1905, proporciona un buen ejemplo. En la teoría se postula que la velocidad de la luz es constante, pero no se presupone ninguna ontología en particular para la luz misma, ni para la materia, ni para la propagación de las interacciones físicas en general. La relatividad especial es compatible tanto con una ontología de partículas que actúan a distancia en el espacio vacío, como con una ontología de campos donde las interacciones se propagan por acción local en un medio físico continuo, como el campo electromagnético. Las leyes de la teoría sólo imponen restricciones 
para las ontologías compatibles con la teoría, pero no determinan ninguna ontología en particular. Así, por ejemplo, es una ley física de la relatividad especial que ninguna interacción puede propagarse de una región a otra del espacio-tiempo con una velocidad mayor que la de la luz en el vacío, que resulta un límite para la transmisión de cualquier señal física. No obstante, esta ley no determina que la relatividad especial sea una teoría de campos, como se afirma a veces. La ley es compatible con una ontología de partículas que actúan a distancia, pero impone la restricción de que las interacciones no pueden ser instantáneas, sino que tienen una velocidad límite.

Así pues, la relación entre teoría y ontología no sólo no es unívoca, sino que es de muchos a muchos: una misma teoría física es compatible con diferentes ontologías y una misma ontología es compatible con diferentes teorías físicas. La búsqueda de la ontología de la mecánica cuántica es, por consiguiente, un problema mal planteado, basado en el falso supuesto de la unicidad de la ontología. En términos puramente filosóficos, debería decirse, entonces, que no existe un único conjunto de hacedores de verdad para cada teoría, sino una multiplicidad de conjuntos diferentes, cada uno de ellos suficiente para la verdad de la teoría. Dicho de otro modo, el mundo podría ser de maneras muy diferentes en caso de que la teoría cuántica estándar fuera verdadera. Ello vale también para cualquier teoría alternativa. Todavía en otros términos, hay muchos mundos físicamente posibles de acuerdo con la mecánica cuántica. Una teoría física no describe un único mundo determinado, sino una pluralidad de mundos posibles. Esta no es más que otra manera de decir que una y la misma teoría (si es satisfacible) siempre tiene una pluralidad de modelos diferentes. Aquella ontología que se considera la ontología de una determinada teoría física no puede ser más que una ontología pretendida, una entre muchas otras posibles.

¿Se sigue de todo esto que la ontología de una teoría es irrelevante? Algunos filósofos, como Quine, los han afirmado de manera explícita. Según su posición:

La referencia y la ontología retroceden, por tanto, al status de meros auxiliares. Las oraciones verdaderas, teóricas y observacionales, son el alfa y el omega de la empresa científica. Están relacionadas por la estructura, y los objetos figuran como meros nodos de la estructura. Qué objetos particulares puedan existir es indiferente para la verdad de las oraciones observacionales, indiferente para el apoyo que éstas otorgan a las oraciones teóricas, indiferente para el éxito de la teoría en sus predicciones. (Quine, 1992: 31) 
Esta conclusión, sin embargo, depende de una concepción general de los fines de la ciencia. Para una posición empirista, los objetivos principales del conocimiento científico son la sistematización de los fenómenos y la predicción de los eventos futuros. Desde este punto de vista, la ontología de una teoría física es un asunto secundario, ya que no afecta a la adecuación empírica de dicha teoría. $\mathrm{Si}$, en cambio, se pretende obtener una imagen global del mundo físico, la ontología pretendida adquiere relevancia. No obstante, ninguna interpretación realista de las teorías empíricas puede reivindicar la idea de que existe algo así como la ontología determinada por una teoría, del mismo modo que, por razones puramente lógicas, no existe el modelo determinado por una teoría. Las teorías restringen la clase de sus posibles modelos y de sus posibles ontologías, pero nunca las determinan.

¿Qué sentido tiene, entonces, la búsqueda de una ontología para la teoría cuántica? Como ya se ha dicho, tal ontología no puede ser más que una ontología pretendida. ¿Para qué sirve disponer de tal ontología? Muchas teorías físicas, incluso cuánticas, no tienen una ontología pretendida, de manera análoga a la que muchas teorías matemáticas no tienen un modelo pretendido. La teoría cuántica estándar, desde sus propios orígenes, careció de tal ontología. Los ejemplos paradigmáticos de entidades cuánticas eran partículas elementales como el electrón y, de manera más o menos implícita, se suponía que el dominio de la teoría lo formaban las entidades microscópicas. La transición del mundo cuántico al mundo clásico siempre constituyó un problema para este punto de vista. Incluso se admitía que la línea de demarcación entre ambos mundos no era nítida, sino un asunto más o menos convencional. Actualmente, esta posición no parece sostenible y la mayoría de los físicos y filósofos de la física considera que la teoría cuántica tiene un alcance universal y que, en particular, se aplica al dominio de las entidades mesoscópicas y macroscópicas. ${ }^{17}$ Sin embargo, no es claro cuáles sean los modelos pretendidos de la teoría, ni cuál la ontología presupuesta en el dominio de estos modelos. En particular, la superposición de estados, que es un elemento esencial de la teoría estándar, impide concebir una ontología coherente, ya que no es posible dar sentido a la noción de una entidad macroscópica en superposición de estados de un observable como la posición. Desde este punto de vista, la ontología de la teoría cuántica estándar siempre ha sido inescrutable, y todavía permanece en ese estado.

${ }^{17}$ Sobre el llamado problema del límite clásico véase Schlosshauer (2007). 


\section{Dos argumentos contra las interpretaciones totales}

Muchos físicos, como ya he señalado, consideran que la interpretación minimal de la mecánica cuántica es insatisfactoria, pero raramente intentan explicar qué condiciones debería satisfacer una interpretación satisfactoria. Roberto Torretti ha señalado con razón que las interpretaciones totales son "aventuras metafísicas" porque consideran el significado y el alcance de la mecánica cuántica desde puntos de vista que son externos a la ciencia empírica (Torretti, 1999: 367). Algunas de estas interpretaciones, señala Torretti, fueron propuestas como alternativas a la teoría estándar, pero no porque hubiera algún resultado experimental incompatible con dicha teoría, sino sobre la base de determinadas preferencias metafísicas. Tal es el caso de la teoría de variables ocultas de Bohm. Otras, como la interpretación de los muchos mundos, al menos en alguna de sus formulaciones, conducen directamente a "una teoría metafísica de la clase menos reputada" (Torretti, 1999: 368).

Un ejemplo más reciente de aventuras que culminan en esta clase de metafísica es el llamado "realismo de la función de onda", propuesto originalmente por David Albert (1996) y desarrollado luego por diferentes filósofos de la física. ${ }^{18}$ Según este punto de vista, las funciones de onda de las cuales habla la teoría cuántica son "objetos físicos concretos" (Albert, 2013: 53), más específicamente, son campos. Pero no se trata de objetos que existan en el espacio tridimensional, sino, directamente en un espacio de un número superior de dimensiones. Albert lo caracteriza diciendo que:

[...] es un espacio que es isomorfo a lo que en las concepciones más convencionales de la mecánica cuántica se hace referencia como el espacio de configuración del mundo, un espacio cuya dimensionalidad es tres veces tan grande como el número total de partículas en el universo. (Albert, 2013: 53, subrayado por el autor)

En principio, hay aquí un equívoco terminológico porque la función de onda es una entidad matemática, un vector, y no un objeto físico. Debería decirse, entonces, que la función de onda se refiere a determinados objetos físicos concretos. ${ }^{19}$ El núcleo de la argumentación consiste en tomar seriamen-

18 La metafísica de la mecánica cuántica propuesta por Albert se discute con mucho detalle en Ney y Albert, (2013).

19 Es preferible llamarla el vector de estado, en vez de la función de onda, para evitar equívocos, pero este último término ya se encuentra establecido por el uso. Siguiendo a Maudlin (2013: 128), conviene decir que la función de onda, en tanto símbolo matemático que tiene una interpretación física, se refiere 
te la existencia del espacio de configuración, que es un espacio puramente matemático, como si fuera un espacio físico. Como se sabe, en la mecánica cuántica la función de onda de un sistema de $N$ partículas se representa en un espacio de $3 N$ dimensiones. Pero usualmente se considera a este espacio como un mero artificio matemático, análogo al espacio de las fases de la física estadística clásica. Albert y sus seguidores, en cambio, lo toman como una representación del espacio físico real. Así pues, según su punto de vista, la mecánica cuántica se refiere a campos en un espacio multidimensional, posiblemente, de infinitas dimensiones. El espacio tridimensional en el cual se desenvuelve nuestra experiencia, por su parte, no es más que una apariencia. Pero, como no conocemos el número de partículas del universo, tampoco podemos saber cuál es el número de dimensiones del espacio físico. Resulta difícil pensar que esta especulación metafísica pueda ser algo más que una extravagancia.

Hay al menos dos argumentos que pueden presentarse contra las interpretaciones totales de esta naturaleza. El primero es una variante del argumento de la subdeterminación de las teorías por la experiencia. De acuerdo con este argumento, todas las interpretaciones totales de la mecánica cuántica deben ser empíricamente equivalentes, pues de otro modo serían teorías alternativas y no meras interpretaciones. Por consiguiente, la experiencia no permite distinguir entre ellas. Si una es empíricamente adecuada o inadecuada, todas las demás también lo serán. La justificación de una interpretación determinada, entonces, se encuentra más allá de la experiencia. Sin embargo, hasta el momento no se han hecho explícitos los criterios que permitirían compararlas y determinar que alguna de ellas es superior a las demás. Por esa razón, no se dispone de ningún criterio definido para sostener que una interpretación es mejor que otra, y todo parece reducirse a una cuestión de preferencias subjetivas por una determinada metafísica.

El segundo argumento apela a la imposibilidad de confirmar las interpretaciones totales. Esta clase de interpretaciones no pueden agregar nuevas consecuencias observacionales a las que permite extraer la interpretación minimal. En consecuencia, no solo son empíricamente equivalentes entre sí, sino también lo son respecto de la interpretación minimal. De hecho, todas las interpretaciones totales tienen en común la interpretación minimal, a la cual añaden una superestructura metafísica. Es razonable admitir, entonces, que

\footnotetext{
al estado cuántico, que es la entidad física representada por el símbolo matemático. Numerosos libros de divulgación o de texto confunden la función de onda con el estado cuántico, o, directamente, la función de onda con una onda física o con un objeto físico.
} 
los resultados experimentales que confirman la mecánica cuántica estándar (que, como se sabe, son numerosísimos) solamente confirman su interpretación minimal. Por principio, la superestructura metafísica no puede ser confirmada por ninguna experiencia.

Mosterín y Torretti resumen esta situación de manera muy clara:

[La mecánica cuántica tiene] un carácter único, sin precedentes en la historia de la física, a saber, que su estructura matemática se interpreta, por así decir, a dos niveles. Por un lado está la interpretación pedestre implícita en cualquier exposición de la mecánica cuántica como teoría física, comprobable en un laboratorio. Según ella, el llamado estado de un sistema cuántico encierra información probabilista sobre las cantidades observables en él, la cual puede extraerse aplicando al representante del estado el operador autoadjunto representativo de la cantidad que interesa observar. Aunque se la suele llamar peyorativamente "interpretación minimalista" y hasta se ha dicho que es "aburrida" [...], esta es de hecho la única interpretación que ponen a prueba y respaldan los tests experimentales de la teoría. Por otro lado, hay un espectro de interpretaciones suplementarias más o menos sofisticadas, incompatibles entre sí [...]. (Mosterín y Torretti, 2010: 390)

Desde este punto de vista, que comparto plenamente, las interpretaciones totales de la mecánica cuántica aparecen como intentos gratuitos de metafísica impuestos sobre una teoría empírica bien confirmada (junto con su interpretación minimal) que no parece requerirlos.

No cabe duda de que la metafísica especulativa ha tenido en ocasiones un papel heurístico positivo sobre la ciencia en general y sobre la física en particular. El atomismo de los antiguos griegos suele ser uno de los ejemplos más citados. Pero la situación de la mecánica cuántica no se adapta a la moraleja de este ejemplo. Una cosa es emplear ciertos supuestos metafísicos para buscar una teoría cuando todavía no se la posee ni se tienen ideas precisas acerca de sus rasgos más generales, como ocurría en la antigüedad, y otra muy distinta es apelar a la especulación metafísica para interpretar una teoría física bien desarrollada y confirmada como la mecánica cuántica estándar. En este último caso no parece haber justificación para el retorno a la especulación metafísica. Por lo demás, cualquier ontología que se pretenda extraer de la mecánica cuántica, o incorporar a ella, no será más que una ontología pretendida entre otras posibles. Ni el formalismo de la teoría ni su interpretación 
minimal determinan una ontología específica. Lo mismo vale para cualquier teoría alternativa a la mecánica cuántica ortodoxa, que siempre será compatible con diferentes ontologías.

\section{Conclusión}

La mecánica cuántica estándar es una teoría interpretada. Su interpretación minimal es ampliamente compartida y resulta suficiente para todas las aplicaciones de la teoría. Cualquier científico o filósofo de la ciencia de orientación anti-realista, en alguna de sus múltiples variedades, debería considerarse satisfecho con esta interpretación. Para los fines de predecir los resultados de posibles experimentos, no se requiere otra interpretación. Por consiguiente, la confirmación de la teoría cuántica tampoco necesita más interpretación que la minimal.

Los filósofos y científicos de orientación realista, sin embargo, no se sienten satisfechos. La interpretación minimal no proporciona una ontología para esa teoría, al menos, no una ontología coherente y completa que pueda considerarse como una imagen global del mundo físico. Las interpretaciones totales pueden entenderse como intentos de proporcionar dicha ontología, pero al hacerlo apelan una especulación metafísica que no puede ser confirmada por ninguna experiencia. Se trata de una clase de metafísica que la filosofía contemporánea ha dejado atrás hace mucho tiempo.

No parece haber ningún criterio preciso para preferir una interpretación total sobre otra, dado que todas son, en principio al menos, lógicamente consistentes y empíricamente adecuadas. Tampoco parece haber ninguna receta para extraer una ontología en particular a partir de un análisis de la estructura de la teoría. El formalismo del espacio de Hilbert no permite ninguna conclusión al respecto, ya que es tan ontológicamente neutral como el cálculo diferencial o la aritmética de los números naturales. No existe ninguna relación necesaria entre el formalismo del espacio de Hilbert y la mecánica cuántica. Esta teoría se formuló originalmente sin ese formalismo y admite todavía otras formulaciones que no lo emplean. Por otra parte, diferentes teorías cuánticas rivales pueden formularse en el espacio de Hilbert. Éste no es más que un instrumento matemático conveniente para la formulación de la mecánica cuántica, que no debe identificarse con ninguna teoría en particular. ${ }^{20}$

\footnotetext{
${ }^{20}$ Adviértase que muchos de los llamados "teoremas de la mecánica cuántica" son en realidad teoremas puramente matemáticos acerca del espacio de Hilbert que, por consiguiente, resultan válidos independientemente de cualquier hipótesis física. Si en el futuro la teoría cuántica estándar fuera refutada por la
} 
Cualquier ontología que se proponga como base de una interpretación total de una teoría física no puede ser más que una ontología pretendida. Ello vale tanto para la mecánica cuántica estándar como para cualquier teoría cuántica alternativa. Todas ellas necesitarán alguna interpretación minimal para poder ser aplicadas y confirmadas por los resultados experimentales, pero serán siempre compatibles con diferentes ontologías globales. Por consiguiente, el problema interpretativo de encontrar una interpretación total de la mecánica cuántica estándar no puede resolverse mediante un cambio de teoría. A lo sumo, lo que podrá obtenerse es una teoría con una ontología pretendida más familiar, más intuitiva o más satisfactoria desde un punto de vista estético, o bien más acorde con las preferencias metafísicas de cada uno. La Mecánica Bohmiana, por ejemplo, surgió como un intento de restaurar una ontología clásica de partículas materiales, pero esa es solo su ontología pretendida, una entre otras posibles, pero no la única que admite la teoría. El problema interpretativo no tiene solución si lo que se pretende es encontrar la ontología determinada por la teoría cuántica. Ese es un seudoproblema que se basa en el falso supuesto de que hay una única ontología posible para cada teoría.

\section{Referencias bibliográficas}

Albert, D. (1992). Quantum Mechanics and Experience. Cambridge, MA: Harvard University Press.

Albert, D. (1996). "Elementary Quantum Metaphysics". En Cushing, J., Fine, A. \& Goldstein, S. (eds.) Bohmian Mechanics and Quantum Theory: An Appraisal. Dordrecht: Kluwer, 1996, pp. 277-284.

Albert, D. (2013). “Wave Function Realism”. En Ney \& Albert (eds.) (2013), pp. 52-57.

Allori, V. (2013). "Primitive Ontology and the Structure of Fundamental Physical Theories". En Ney \& Albert (eds.) (2013), pp. 58-75.

Auletta, G., Fortunato, M. \& Parisi, G. (2009). Quantum Mechanics. New York: Cambridge University Press.

Auletta, G. \& Wang, S. W. (2014). Quantum Mechanics for Thinkers. Singapore: Pan Stanford Publishing. 
Bacciagaluppi, G. \& Valentini, A. (2009). Quantum Theory at the Crossroads: Reconsidering the 1927 Solvay Conference. New York: Cambridge University Press.

Bitвol, M. (1996). Mécanique quantique. Une introduction philosophique. Paris: Flammarion.

Boнm, D. (1951). Quantum Theory. New York: Prentice-Hall. (Reimpreso en New York: Dover, 1989).

Bонм, D. (1952). "A Suggested Interpretation of Quantum Theory in Terms of Hidden Variables". Physical Review, 85: 166-193.

Boнm, D. (1957). Causality and Chance in Modern Physics. London: Routledge.

Bohm, D. \& Hiley, B. (1993). The Undivided Universe: An Ontological Interpretation of Quantum Theory. London: Routledge.

BuB, J. (1999). Interpreting the Quantum World. Revised Edition. Cambridge : Cambridge University Press.

Bub, J. (2007). “Quantum Probabilities as Degrees of Belief'. Studies in History and Philosophy of Modern Physics, 38: 232-254.

CAssini, A. (2016). "La paradoja de Einstein-de Broglie. ¿Incompletitud o superposición de estados?". Cadernos de História e Filosofia da Ciência. [En prensa].

Cohen-Tannoudj, Diu, B. \& Lalö̈, F. (1977). Quantum Mechanics. New York: Wiley.

Cushing, P. (1998). Philosophical Concepts in Physics: The Historical Relation between Philosophy and Scientific Theories. Cambridge: Cambridge University Press.

Dickson, W. M. (1998). Quantum Chance and Non-Locality: Probability and Non-Locality in the Interpretations of Quantum Mechanics. Cambridge: Cambridge University Press.

DiRAC, P. (1967). The Principles of Quantum Mechanics. Fourth Edition. Oxford: Clarendon Press. [1a ed. 1930].

Dürr, D., Goldstein, S. \& Zanghi, N. (2013): Quantum Physiscs without Quantum Philosophy. Berlin: Springer.

DürR, D. \& Teufel, S. (2009). Bohmian Mechanics: The Physics and Mathematics of Quantum Theory. Berlin: Springer. 
FrIedRICHS, S. (2015). Interpreting Quantum Theory: A Therapeutic Approach. New York: Palgrave Macmillan.

Fuchs, C. \& Peres, A. (2000). "Quantum Theory Needs No 'Interpretation"”. Physics Today, 53: 70-71.

Ghirardi, G. (2005). Sneaking a Look at God's Cards: Unraveling the Mysteries of Quantum Mechanics. Princeton: Princeton University Press.

Ghirardi, G. Rimini, A. \& Weber, T. (1986). "Unified Dynamics for Microscopic and Macroscopic Systems". Physical Review, D34: 470-491.

GIERE, R. (1988). Explaining Science: A Cognitive Approach. Chicago: The University of Chicago Press.

Gouesbet, G. (2013). Hidden Worlds of Quantum Physics. New York: Dover.

Heisenberg, W. (1958). Physik und Philosophie. München: Piper.

Holland, P. (1993). The Quantum Theory of Motion. Cambridge: Cambridge University Press.

Howard, D. (2004). "Who Invented the 'Copenhaguen Interpretation'? A Study in Mythology" Philosophy of Science, 71: 669-682.

Isham, C. (1995). Lectures on Quantum Theory: Mathematical and Structural Foundations. London: Imperial College Press.

JAEGER, G. (2009). Entanglement, Information, and the Interpretation of Quantum Mechanics. Berlin: Springer.

JAEger, G. (2014). Quantum Objects: Non-Local Correlation, Causality, and Objective Indefiniteness in the Quantum World. Berlin: Springer.

Jammer, M. (1974). The Philosophy of Quantum Mechanics: The Interpretations of Quantum Mechanics in Historical Perspective. New York: John Wiley.

LALOË, F. (2012). Do We Really Understand Quantum Mechanics? New York: Cambridge University Press.

Maudlin, T. (2013). "The Nature of the Quantum State". En Ney \& Albert (eds.) (2013), pp. 126-153.

Mermin, D. (2004). "What's Wrong with this Quantum World". Physics Today, 57: 10-11.

Mosterín, J. \& Torretti, R. (2010). Diccionario de Lógica y Filosofia de la Ciencia. Segunda edición. Madrid: Alianza. 
Ney, A. \& Albert, D. (eds.) (2013): The Wave Function: Essays on the Metaphysics of Quantum Mechanics. New York: Oxford University Press.

OMnès, R. (1994). The Interpretation of Quantum Mechanics. Princeton: Princeton University Press.

OMnÈs, R. (1999). Understanding Quantum Mechanics. Princeton: Princeton University Press.

Peres, A. (2002). Quantum Theory: Concepts and Methods. New York: Kluwer.

Petersen, A. (1963). "The Philosophy of Niels Bohr". Bulletin of the Atomic Scientists, 19: 8- 14.

Petersen, A. (1968). Quantum Mechanics and the Philosophical Tradition. Cambridge, MA: The MIT Press.

Quine, W. V. O. (1968). "Ontological Relativity”. The Journal of Philosophy, 65: $185-212$.

Quine, W. V. O. (1992). Pursuit of Truth. Revised Edition. Cambridge, MA: Harvard University Press.

Riggs, P. (2009). Quantum Causality: Conceptual Issues in the Causal Theory of Quantum Mechanics. Dordrecht: Springer.

Ruetsche, L. (2011). Interpreting Quantum Theories. New York: Oxford University Press.

Schlosshauer, M. (2007). Decoherence and the Quantum-to-Classical Transition. Berlin: Springer.

Selleri, F. (1994). Le grand débat de la théorie quantique. Paris: Flammarion.

Torretti, R. (1999). The Philosophy of Physics. Cambridge: Cambridge University Press.

Van Fraassen, B. (1991). Quantum Mechanics: An Empiricist Approach. Oxford: Clarendon Press.

Von Neumann, J. (1932). Mathematische Grundlagen der Quantenmechanik. Berlin: Springer.

Weinberg, S. (2013). Lectures on Quantum Mechanics. New York: Cambridge University Press. 